\title{
PREFACIO
}

\section{LA PROFESIONALIZACIÓN DE LA ACADEMIA EN CHILE}

\section{Andrés Bernasconi*}

Este 2008 se cumplen 40 años de varios hitos significativos en la historia de la universidad. Masivas protestas de estudiantes en París, Varsovia, Madrid, Nueva York, Berkeley y Buenos Aires, entre otros centros académicos, redefinieron el rol de los estudiantes en el gobierno universitario y señalaron la irrupción en la vida política de una nueva generación. La matanza de Tlatelolco, en ciudad de México, expresó de manera trágica la incapacidad de los gobiernos para reaccionar ante las demandas de este nuevo actor político por medios distintos a la represión. Nuestra propia reforma universitaria trajo a los claustros de las universidades chilenas las banderas de cambio agitadas por primera vez en Córdoba medio siglo antes. No en vano la prensa internacional ha recordado 1968 como el año que definió quiénes somos: los jóvenes universitarios de entonces dirigen nuestras sociedades hoy.

Paradoja exquisita de la historia es que el mismo año en que los estudiantes ponían sitio a la "torre de marfil" de profesores olímpicamente desentendidos de los cambios sociales que hervían bajo sus narices, se publicara la crónica definitiva del ascenso de los académicos al control de las universidades en EE.UU. En efecto, en 1968 apareció la primera edición de "The Academic Revolution", el clásico de Christopher Jencks y David Riesman ${ }^{1}$ sobre el proceso de profesionalización de la academia y el inexorable incremento de la influencia de los profesores sobre las universidades que resultó de dicha profesionalización.

* Universidad Andrés Bello.

1 Hay traducción al castellano por Paidós, 1970: La Revolución Académica. 
La historia que cuentan Jencks y Riesman tiene cierta relevancia para Chile. Como en América Latina, y a diferencia de Europa, el desarrollo de la educación superior en EE.UU. es un fenómeno que en verdad se inicia el siglo XIX, no obstante los precedentes de colleges de inspiración religiosa que, al igual que las universidades reales y pontificias en América hispana, datan de la era colonial. Así como los colleges de EE.UU. se organizaron desde el siglo XVII (en la medida que las pobres condiciones locales permitían) según el modelo de Oxbridge, los de América hispana procuraron imitar los ejemplos de Salamanca y Alcalá, con semejantes escasos resultados. Si bien la independencia de EE.UU. precedió por medio siglo la de las colonias del Sur, sólo a partir de la Era de Jackson (fines de los años de 1820) empiezan los Estados Unidos de América a separarse culturalmente del imperio británico, época que coincide con la de la independencia política de los antiguos dominios españoles.

Pero sólo hasta aquí llegan las semejanzas. Mientras durante la primera mitad del siglo XIX en América del Norte florecen cientos de colleges que expresan el pluralismo de convicciones religiosas, raza, sexo, ocupaciones profesionales, clases sociales y ambientes regionales de sus fundadores y las comunidades en que se ubican, en América Latina surge la "Universidad Nacional" con sus aspiraciones racionalistas de orden y progreso guiadas por la iniciativa iluminista del estado docente. Mientras en Norteamérica en el siglo XIX las universidades son gobernadas por los trustees que se sientan en sus juntas directivas, en el Sur es el gobierno central el que rige sus destinos. Mientras el cultivo científico de las disciplinas comienza en EE.UU. en los años de 1880-1890 en Harvard, Johns Hopkins, Yale, Chicago y Columbia, en la mayor parte de nuestra región (Argentina es una excepción) la investigación -y sólo en las ciencias naturales y exactas- es un fenómeno de la segunda mitad del siglo XX.

Con todo, la divergencia de mayor interés aquí es de data algo más cercana y explica buena parte de las diferencias entre las universidades de Norteamérica y las del sur del Río Grande. Nos referimos, desde luego, a la profesionalización del profesor 
universitario, que se inicia en EE.UU. en la primera mitad del siglo XX y se completa hacia la década del 50, mientras que en nuestros países -con excepción de una docena de universidades en Brasil- todavía no culmina.

Por profesionalización, Jencks y Riesman entienden la transformación del profesor en un experto independiente, entrenado en investigación (generalmente a través de un doctorado), con dedicación completa a la actividad académica y que genera conocimiento según estándares de desempeño establecidos y controlados por sus pares. En palabras de los autores:

"Un gran número de PhDs actualmente se ven a sí mismos como profesionales independientes -como médicos o abogados- responsables primariamente ante ellos mismos y sus colegas antes que a sus empleadores, y comprometidos con el avance del conocimiento antes que el de una institución en particular (p. 14)."2

Los profesores pasan a controlar el acceso al ejercicio de la profesión a través de un proceso de formación (el doctorado), normado y dirigido por sus miembros ya establecidos. Asimismo, instauran y vigilan en forma exclusiva las normas de buena práctica del oficio por medio de la institución de la evaluación por pares, a la que se recurre cada vez que es necesario emitir juicio sobre la idoneidad profesional de un colega, ya sea para efectos de la contratación, la calificación, el desempeño o la promoción en la carrera académica, la que también es definida y cautelada por los mismos miembros de la academia. Finalmente, lo que cuenta como conocimiento válido es delimitado por los académicos actuando como editores de revistas científicas y árbitros de los proyectos de investigación y publicaciones de sus colegas.

2 Las referencias son a la edición de 2001, publicada por Transaction (New Brunswick), y la traducción es mía. 
Jencks y Riesman no se detienen en la constatación de estos fenómenos, sino que examinan sus consecuencias para el funcionamiento de las universidades. Así, por ejemplo, la profesión académica adquiere las prerrogativas de fijar los estándares de admisión de los estudiantes al posgrado y de incidir en los criterios de ingreso al pregrado, diseñar el currículum, definir el contenido de los cursos, determinar los libros de texto, escoger a los colegas que enseñarán, definir los departamentos en que se estructura la actividad académica y elegir de entre ellos a las autoridades departamentales.

El poder del académico profesional no se restringe al departamento: The Academic Revolution plantea que las juntas directivas (boards) de las universidades han pasado a tener una función crecientemente ceremonial, en la medida en que el gobierno institucional recae hoy en administradores profesionales. Pero estos altos directivos universitarios gobiernan no en interés del directorio sino en el de los profesores, toda vez que la principal preocupación de los líderes universitarios es atraer y mantener en sus instituciones a académicos eminentes. El académico profesional es sólo relativamente dependiente de la universidad que lo emplea, por cuanto tiene la capacidad de ganar recursos para su trabajo y de moverse con ellos a la institución que elija. Así, cuando un rector habla de fortalecer su institución, lo que está diciendo, a fin de cuentas, es que quiere mantener en ella a los profesores más destacados y atraer a otros tantos. No obstante que los profesores suelen ver a "la administración" como el enemigo (ver a propósito, en este volumen, el artículo de Enrique Fernández) y a descargar contra ella la propensión a la irritabilidad que los caracteriza, la verdad es que el profesorado es el estamento al que la administración dedica mayor atención y cuidado. Ésta sólo tiene cierto margen de acción autónoma en los asuntos respecto de los cuales los profesores están divididos.

La profesión académica, así entendida, llega a América Latina sólo recientemente y está todavía lejos de constituir el modo dominante de ser académico. Lo que Jesús Galaz y sus coautores describen sobre México en esta edición es generalmente aplicable a toda la región: la 
expansión de la matrícula a partir de los años 70 obligó a improvisar académicos de entre las filas de los recién licenciados, quienes carecieron de una formación de posgrado rigurosa y dedicaron su vida académica a la docencia. Los centros de investigación que en la segunda mitad del siglo XX se crearon en algunos países de la región, dentro y fuera de la universidad, fueron durante décadas iniciativas marginales respecto de la orientación global de la educación superior hacia el entrenamiento de profesionales.

Chile no es excepción y se encuentra aún en transición desde una academia amateur a una profesional. La amateur -uso la expresión como lo opuesto a profesional y sin intención peyorativa- es la de los profesores docentes, sean éstos profesionales que tienen su práctica fuera de ella y enseñan algunos cursos, o empleados de tiempo completo en la universidad. La academia basada en el profesional de ejercicio liberal tiene hondas raíces en la historia y tradición de la universidad latinoamericana y dio frutos valiosos en Chile durante todo el siglo XIX y buena parte del XX. Distinguidos hombres de letras que, salvo notables excepciones, no vivían en o de la universidad, formaron las profesiones, organizaron el Estado y difundieron la educación a lo largo del país (Mellafe et al., 1992; Serrano, 1994). Abogados, médicos e ingenieros destacados formaron generaciones de profesionales que dieron renombre a la universidad chilena en el concierto regional.

Pero ese modelo de universidad no podía durar ante los cambios que experimentaba la sociedad y la relación entre ella y el conocimiento. La crisis de la academia amateur se manifestó en la reforma universitaria de fines de la década de los 60. Entre otras demandas, los reformistas buscaban jornadas completas y una carrera que permitieran expandir las funciones de la universidad hacia la investigación y la extensión. La reforma habría sido imposible sin el desarrollo sistemático de la investigación en las ciencias naturales y exactas y en las humanidades, que comienza tímidamente en la década de 1960: profesores de tiempo completo, muchos de ellos formados en el extranjero, reclamaban mayores espacios para desarrollar una labor académica integral (Brunner, 1986). 
Podríamos quizás decir, sin forzar demasiado las efemérides, que la profesionalización de la academia comienza en Chile justo hace 40 años, con la reforma, el mismo año en que dicho proceso se proclama culminado en EE.UU. En Chile ha sido éste un proceso lento que sólo ha terminado en la profesionalización completa de los departamentos de ciencias naturales y exactas en un par de universidades, conformándose así unidades académicas integradas sólo por doctores activos en investigación (Bernasconi, 2005). La profesionalización de la academia en las ciencias sociales, las humanidades y las profesiones es aún menos generalizada (Bernasconi, 2007). Sólo en las últimas dos décadas el doctorado se ha erigido en requisito sine qua non de ingreso a la profesión académica en algunos departamentos en las universidades de mayor dedicación a la investigación. La norma de sólo contratar doctores como una política general a lo largo y ancho de toda una universidad es aún más reciente (ver el trabajo de Juan José Ugarte, Bárbara Loeb y Mauricio Ferrari en este número).

A esta profesionalización han contribuido variados factores, entre ellos, la influencia del modelo de la research university de EE.UU. (Bernasconi, 2008), el aumento de los salarios de los profesores hasta un nivel que permite una dedicación completa a la universidad, la necesidad de diferenciarse que impone la competencia en el mercado de la educación superior (ver el artículo de Paulina Berríos, en este volumen) y las políticas de investigación, tanto del gobierno como de las universidades (ajustadas con impecable racionalidad a aquéllas, fuente de los recursos) que en los últimos 20 años han venido definiendo y reforzando una métrica de logro científico basada en productos estandarizados. Como sugieren los trabajos de Fernando Lolas y Paulina Berríos en esta edición, los atributos de validez y objetividad con que dicha métrica aparece revestida explican en parte el predominio de las labores de investigación en la definición de las funciones que se esperan de un profesor universitario: se evalúa lo que se puede medir.

Algunas observaciones de Jencks y Riesman suenan tan cercanas que parecen haberse escrito a propósito de la academia 
chilena. Señalan los autores que, como el volumen de recursos crece más rápido que el de investigadores competentes -pensemos en la quintuplicación de los recursos para ciencia desde 1990, y la harto menos espectacular expansión del parque erudito-, los investigadores de mayor talento son escasos y han aumentado sus salarios. A ello podemos agregar la competencia feroz entre las universidades por reclutar ese talento, aunque haya que levantarlo desde la universidad vecina. Especulan estos autores que a ello seguiría la rebaja en la carga docente de pregrado para estos profesores, la concentración de sus esfuerzos en estudiantes de posgrado y la eliminación de profesores incapaces de trabajo académico de alto nivel, ejemplos de lo cual no faltan en Chile, como atestigua el trabajo de Enrique Fernández en este número.

Nótese que la profesionalización a la que aludo se erige en torno a la investigación y, básicamente, ignora la docencia. La enseñanza no es el foco de la educación doctoral, los pares escasamente evalúan la docencia y no hay estándares universalistas que permitan juzgar quién es quién en esta la más antigua y permanente función universitaria. Es posible que la falta de profesionalización de la enseñanza sea corregida en el futuro, creándose rigurosos programas de formación docente, mecanismos de evaluación por pares y mediciones objetivas de desempeño instruccional. Se habrá creado, entonces, una profesión académica docente equivalente a la de investigación, o a la de gestión, que también se profesionaliza en la medida en que los cargos superiores de la administración universitaria son servidos por especialistas dedicados a ello. Mientras tanto, un profesor con dedicación completa a la enseñanza, como los que abundan en las universidades chilenas menos involucradas en investigación, no es un académico profesional, en el sentido que he usado aquí. Esto no equivale a decir que su labor no sea importante y meritoria. En efecto, el trabajo de Enrique Fernández en este número señala que la docencia es el elemento menos dispensable en los compromisos de desempeño académico que los profesores pactan con sus jefes de unidad en cada vez más universidades del país. 
La noción del profesor universitario como una suerte de profesional liberal, que se identifica antes con su disciplina que con la institución en que trabaja, es en alguna medida desvirtuada por el sindicalismo académico, fenómeno de cuya aparición en la Universidad Austral de Chile da cuenta el testimonio de Marcela Méndez en este volumen. El sindicato de profesores centra la identidad del académico en su relación de empleo con la universidad, que en cuanto tal es equivalente a la de los funcionarios administrativos, pasando la identidad profesional al segundo plano. Es posible que el sindicalismo capture principalmente a los que Schwartzman (1993: 11) y Jesús Galaz y coautores (en el artículo sobre México en este número) llaman "docentes universitarios" y no a los profesores investigadores. Los docentes universitarios no son los profesionales liberales del Derecho o la Medicina que enseñan a tiempo parcial -los "catedráticos", en la designación de Jesús Galaz y sus colaboradores- ni son investigadores que concentran su atención en sus carreras científicas. Son instructores de tiempo completo de las universidades que llenan la mayor parte de su tiempo en la enseñanza, la administración y la política interna. Por lo dicho, no llama la atención que el sindicato de profesores de la Universidad Austral de Chile se denomine "sindicato de docentes". Los docentes han sido en otros países de América Latina el principal campo de reclutamiento para los sindicatos universitarios y dudo que en Chile vaya a ser diferente. De hecho, como Méndez explica en su testimonio, las asociaciones de académicos cumplen hace años en muchas universidades chilenas las funciones de un sindicato, sin las formalidades de tal.

Por otra parte, que se establezca un estándar de desempeño profesional en torno a la investigación es una cosa, pero que el perfil y las actividades de quienes se desempeñan en nuestras universidades se ajuste a ese patrón es cosa totalmente distinta. En principio, los patrones de lo que debe ser un académico de excelencia se expresan formalmente en las normas que rigen la vida académica en la universidad y, en particular, como pone de relieve el artículo de Paulina Berríos, en los reglamentos de carrera académica. Sin embargo, la variedad de oficios académicos que coexisten en las universidades 
de nuestro país y las tensiones que ello genera son hechos bien establecidos, como lo reflejan los trabajos reunidos en este número. Esta diversidad pone un desafío grande frente a los administradores que deben establecer las reglas y políticas que rigen la carrera académica. Los casos publicados en esta revista sobre la implementación de la jerarquización y la evaluación en la Universidad Diego Portales (UDP) y la formulación de una nueva política de gestión del cuerpo académico en la Pontificia Universidad Católica de Chile (PUC) dan cuenta de dichas complejidades. En el caso de la UDP, como explica María José Ramírez, la jerarquización buscó armonizar la historia y valores de la universidad con lo que dichas instituciones chilenas y la comunidad internacional generalmente asocia a las calidades de profesor titular, asociado e instructor. El texto de Juan José Ugarte, Bárbara Loeb y Mauricio Ferrari sobre la experiencia de la PUC, por su parte, ilustra el esfuerzo por alinear "la diversidad de vocaciones e intereses y, por tanto, distintas posibilidades de avanzar en la carrera académica", con el principio de que la PUC es una universidad donde "se enseña lo que se investiga". Esta tensión se resuelve, en el caso de la PUC, con la adscripción de los profesores investigadores a la carrera "ordinaria", en tanto que los profesores que no combinan docencia e investigación quedan ubicados en la carrera "especial".

Los casos de la UDP y la PUC aquí presentados dan sustento a la tesis de Paulina Berríos de que en nuestro país la definición de la carrera académica resulta de "una relación dialéctica" entre las funciones que los miembros del cuerpo académico efectivamente cumplen, por un lado, y las expectativas normativas de los directivos superiores de las universidades, por otro. Los compromisos de desempeño y su evaluación, de que trata el artículo de Enrique Fernández, son otra formalización más de esa relación binaria. A los profesores y directivos como modeladores de la carrera académica podemos agregar todavía al Estado que, como argumentan Jesús Galaz y sus coautores, comentando las experiencias del Sistema Nacional de Investigadores y del Programa para el Mejoramiento del Profesorado en México, puede tener un impacto decisivo en los roles de los académicos. En esto las experiencias aquí retratadas se apartan 
del origen exclusivamente "de abajo hacia arriba" que, en concepto de Jencks y Riesman, caracteriza la carrera académica en un entorno enteramente profesionalizado.

Es cierto que en el modelo de gobierno de las universidades de América Latina los profesores son también los directivos de las instituciones, y por esta razón podría pensarse que la carrera académica es definida exclusivamente por profesores. En efecto, las universidades públicas carecen por regla general de directorios formados por personalidades ajenas a la universidad, y todas las autoridades unipersonales y colegiadas son elegidas mayoritariamente por profesores. Así, el control de éstos sobre la organización es tan completo como el que describen Jencks y Riesman para la universidad estadounidense. Sin embargo, los déficit en la profesionalización de los académicos, los remanentes de cogobierno estudiantil que subsisten en la mayoría de los países de la región y la casi total dependencia de financiamiento gubernamental discrecionalmente asignado tienden a politizar la naturaleza de ese control: ante la ausencia o debilidad de un ethos científico y de estándares académicos por los cuales velar, el gobierno de las universidades se consume, en lo externo, en cortejar el favor ministerial, y en lo interno en alcanzar y conservar el poder por el poder. La carrera académica definida en este contexto no puede sino reflejar el resultado del peso político de las distintas facciones universitarias.

En las universidades privadas, por su parte, el control radica no en los profesores, sino en los propietarios, sean éstos iglesias, empresarios o entidades filantrópicas. Con todo, la magnitud del contrapeso que representan los profesores depende precisamente del grado de profesionalización de la academia. Allí donde las universidades emplean sólo o predominantemente instructores de tiempo parcial para hacer docencia y no existen cuadros permanentes para investigación, los profesores tienen un peso insignificante en el gobierno, pero cuando los académicos profesionales pasan a ser mayoría, el gobierno institucional tiende a gravitar hacia la agenda de los profesores. 
La influencia de los académicos no se agota en las universidades: crecientemente los profesores son llamados a participar como especialistas en el diseño, implementación y evaluación de políticas públicas, son las principales voces especialistas en el debate público, actúan como consultores en los más diversos proyectos de la iniciativa privada y pública y contribuyen a las artes y la cultura con sus creaciones.

En el caso de las políticas públicas, cuando se analiza la experiencia de los últimos 30 años de la educación superior chilena, en términos de Estado vs. mercado o viceversa, se suele ignorar o minimizar la influencia de los profesores, que frecuentemente actúan como bisagra o amortiguador entre esos otros dos polos de coordinación del sistema. Piénsese, por ejemplo, que entre un cuarto y un tercio del presupuesto de educación superior y ciencia en Chile es distribuido sobre la base de competencia de proyectos evaluados, arbitrados y a veces también directamente adjudicados por académicos. Me refiero, desde luego, a los fondos concursables de investigación y becas de posgrado, pero a ello se suman también los proyectos de desarrollo institucional tipo Programa de Mejoramiento de la Calidad y Equidad de la Educación Superior (Mecesup), Fondo de Desarrollo Institucional (FDI) y convenios de desempeño. Aunque estos dineros no son el grueso del financiamiento a las universidades, sin embargo, ellos son precisamente los recursos incrementales que no están amarrados a fórmulas o criterios históricos de distribución y que permiten a las universidades hacer algo más que subsistir.

Son académicos también quienes componen todos los órganos formales de gobierno de la educación superior chilena: los consejos de la Comisión Nacional de Investigación Científica y Tecnológica (CONICYT), el Consejo Superior de Educación (CSE), la Comisión Nacional de Acreditación (CNA) y el Consejo de Rectores de las Universidades Chilenas (CRUCH). No es casualidad que desde 1990 todos los jefes de la División de Educación Superior del Ministerio del ramo han sido profesores universitarios. 
Y si es cierto que las universidades son entidades maximizadoras de prestigio, como suele decirse, nuevamente los académicos tienen algo que decir, y no sólo por la razón evidente de que el prestigio de una universidad es el de sus profesores. Me refiero a la labor de regulación de prestigios que desde el Estado ejercen los profesores, actuando como pares evaluadores. La acreditación, que se basa en el juicio de pares académicos, corona o destruye reputaciones según los dictámenes que emite y las dosis de años de acreditación que administra.

Desde la esquina del mercado, por su parte, los rankings, cuando están razonablemente bien hechos, descansan sobre la misma métrica de actividades de investigación que constituye a la profesión académica, complementada por la opinión de profesores sobre cuáles son los mejores programas y las mejores universidades, juicio que, a fin de cuentas, es el único más o menos informado de que disponemos en estas materias, más bien oscuras para el ciudadano común.

De lo dicho, espero, quedará justificada la opción de los editores de publicar un número de Calidad en la Educación dedicado a la profesión académica en Chile, iniciativa que no cabe sino aplaudir. Este volumen reúne contribuciones que reflejan la investigación que sobre los académicos se está realizando en Chile y en otros países de la región, y da cuenta de los desarrollos más recientes en la relación entre profesores y universidades, y entre aquellos y la sociedad en general.

\section{Referencias bibliográficas}

Bernasconi, Andrés (2008) Is there a Latin American model of the university? Comparative Education Review, 52(1), pp. 27-52.

Bernasconi, Andrés (2007) Are there research universities in Chile? In Philip G. Altbach and Jorge Balán (eds.) World Class Worldwide: Transforming Research Universities in Asia and Latin America. Baltimore: Johns Hopkins University Press. 
Bernasconi, Andrés (2005) University entrepreneurship in a developing country: the case of the Pontificia Universidad Católica de Chile: 1985-2000. Higher Education, 50(2), pp. 247-274.

Brunner, José Joaquín (1986) Informe sobre la educación superior en Chile. Santiago de Chile: FLACSO.

Mellafe, Rolando; Rebolledo, Antonia y Cárdenas, Mario (1992) Historia de la Universidad de Chile. Santiago de Chile: Ediciones de la Universidad de Chile.

Schwartzman, Simon (1993) Policies for higher education in Latin America: the context. Higher Education, 24(1), pp. 9-20.

Serrano, Sol (1994) Universidad y Nación: Chile en el siglo XIX. Santiago de Chile: Editorial Universitaria. 